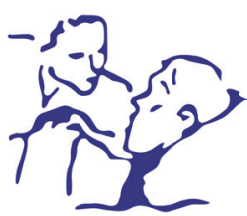

NOTA CLÍNICA

\title{
Naloxegol para el tratamiento del estreñimiento inducido por opioides: un estudio descriptivo de seguimiento anual en tres pacientes con dolor crónico por cáncer
}

\author{
Borja Mugabure Bujedo* \\ Departamento de Anestesiología, Reanimación y Medicina del Dolor, Hospital Universitario, Donostia
}

Recibido el 12 de marzo de 2018

Aceptado el 5 de junio de 2018

\section{PALABRAS CLAVE}

Naloxegol, estreñimiento, opioides, cáncer, dolor.

\section{Resumen}

En este estudio descriptivo de seguimiento de un año en tres pacientes con estreñimiento inducido por el tratamiento con opioides de diversas etiologías de dolor por cáncer, en el contexto de mieloma múltiple, neoplasia de mama y pulmón, hemos observado el buen perfil de eficacia y tolerabilidad del naloxegol oral 12,5-25 mg. Los resultados más relevantes fueron una mejor eficacia del tratamiento en comparación con las opciones anteriores utilizadas, como el ejercicio, la dieta y los laxantes, y también una gran satisfacción personal.

\section{KEYWORDS}

Naloxegol, constipation, opioids, cancer, pain.

\section{Abstract}

In this descriptive, one-year follow-up study of three patients on opioids for pain related to multiple myeloma, breast cancer and lung cancer, who had opioid-induced constipation, we found that oral naloxegol dosed at $12.5-25 \mathrm{mg}$ is highly effective with a good tolerability profile. Most relevant results included higher effectiveness as compared to previous options such as exercise, diet and laxatives, as well as great personal satisfaction.

Mugabure Bujedo B. Naloxegol para el tratamiento del estreñimiento inducido por opioides: un estudio descriptivo de seguimiento anual en tres pacientes con dolor crónico por cáncer. Med Paliat. 2019;26(2):174-178.

\footnotetext{
*Autor para correspondencia:

Borja Mugabure Bujedo

Departamento de Anestesiología, Reanimación y Medicina del Dolor. Hospital Universitario de Donostia.

Paseo del Dr. Beguiristain 107-111. 20014. San Sebastián

correo electrónico: mugabure@yahoo.es
} 


\section{Introducción}

Se estima que la prevalencia del estreñimiento inducido por opioides (EIO) es del $52 \%$ de las personas con neoplasias malignas avanzadas, y esta cifra se eleva al $87 \%$ para las personas con enfermedad terminal en tratamiento con opioides. Aunque la mayoría de los datos provienen de estudios en pacientes con cáncer, el 40-64\% de los pacientes tratados con opioides para el dolor crónico no oncológico padecen EIO a pesar del uso de laxantes ${ }^{1}$.

Los receptores opioides se expresan a lo largo del tracto gastrointestinal y están involucrados en una matriz de múltiples funciones celulares. Este hecho causa cambios que provocan la desaceleración de la motilidad, el aumento del tono esfínteres y la motilidad descoordinada. La magnitud global de los síntomas se denomina disfunción intestinal inducida por opioides (DIIO) (Tabla I).

El EIO es uno de sus muchos síntomas y probablemente el más frecuente y desagradable en estos pacientes. Sus criterios diagnósticos se definen en la Tabla II². Los agonistas opioides causan DIIO, principalmente al unirse a receptores opioides submucosos intestinales. Por lo tanto, los antagonistas opioides que bloquean solo a estos receptores opioides $\mu$ periféricos deben reducir la DIIO o el EIO sin disminuir la analgesia opioide central. Los fármacos denominados antagonistas periféricos del receptor $\mu$ opioide, traducción del acrónimo en lengua inglesa PAMORA (peripherally-acting mu-opioid receptor antagonists), se han evaluado recientemente en ensayos clínicos y están disponibles en algunos países. Entre ellos se encuentran los siguientes: metilnaltrexona, naloxegol, alvimopán y naldemedina. En España, el naloxegol es el único fármaco oral disponible de este grupo, que está en el mercado europeo desde marzo de $2016^{3}$. En nuestro país se ha aprobado con la indicación de pacientes con EIO en el ámbito de enfermedades oncológicas y requiere visado sanitario.

El EIO es un cuadro clínico subdiagnosticado. En los estudios de prevalencia, el estreñimiento ocurre en el $11 \%$ de los pacientes de la población general, mientras que la frecuencia de estreñimiento oscila entre el $33 \%$ y el $94 \%$ en los pacientes tratados con opioides para dolor no oncológico y oncológico, respectivamente ${ }^{4}$. En la encuesta sobre pacientes de efectos relacionados con los opioides (Estudio PROBE) para dolor crónico llevada a cabo en los Estados
Unidos de Norte América y la Unión Europea, el 33 \% declaró que omitió dosis, disminuyó la cantidad del fármaco o suspendió la medicación opioide para aliviar los problemas relacionados con efectos secundarios digestivos. El $92 \%$ de los pacientes experimentó posteriormente un aumento del dolor, de los que el 86 \% informó que disminuyó su calidad de vida y las actividades diarias ${ }^{5}$. De hecho, la carga económica del estreñimiento es sustancial cuanto a los costes directos e indirectos asociados. Los costes directos incluyen visitas médicas, hospitalización, procedimientos diagnósticos y medicamentos. Los costes indirectos incluyen automedicación, pérdida de ingresos, actividad laboral reducida y gastos asociados a los cuidadores. Los pacientes con estreñimiento grave mostraron los costes totales más altos, calculados en 1525 euros por paciente al mes, mientras que los casos de pacientes con problemas leves y moderados supusieron un gasto de 1196 euros y 1088 euros, respectivamente. El coste indirecto debido a la baja por enfermedad fue el elemento de coste más sustancial en todos los grupos ${ }^{6}$.

En este artículo presentamos un estudio descriptivo de 1 año de seguimiento de la eficacia o la tolerabilidad del naloxegol oral en tres pacientes que presentaban EIO para el dolor crónico por cáncer de diversas etiologías.

\section{Casos clínicos}

Reclutamos pacientes con cáncer que fueron remitidos a la Unidad de Dolor Crónico en diciembre de $2016(n=3)$, y se realizó un seguimiento de un año hasta diciembre de 2017.

1. Los pacientes seleccionados deberían estar recibiendo un tratamiento con opioides orales de un mínimo de $30 \mathrm{mg} /$ día de dosis equivalentes de morfina oral, para tratar el dolor relacionado con el cáncer durante al menos 2 semanas antes de la inclusión. No se debía realizar ningún cambio en la dosis de opioides durante la duración del estudio. Los pacientes debían presentar síntomas definidos como ElO durante al menos 3 meses y, además, debían tener dolor basal bien controlado por opioides.

2. Todos los pacientes debían tener un índice de función intestinal (IFI) que obtuviera una puntuación mayor a 30. El IFI es un cuestionario subjetivo, administrado

Tabla I. Mecanismos farmacológicos y síntomas clínicos de la disfunción intestinal inducida por opioides ${ }^{3}$

\begin{tabular}{ll}
\hline \multicolumn{1}{c}{ Mecanismos farmacológicos } & \multicolumn{1}{c}{ Síntomas clínicos } \\
\hline Disminución de la producción de saliva & Xerostomía \\
Dismotilidad del esfínter esofágico inferior & Reflujo gastroesofágico \\
Disminución de la secreción gástrica y su motilidad & Dolor abdominal y retraso del vaciado \\
Disminución de la secreción intestinal & Estreñimiento inducido por opioides, heces duras y secas \\
Contracciones intestinales anormales & Distensión y dolor abdominal espástico \\
Contracción del esfínter de Oddi & Cólico biliar \\
Aumento de tono del esfínter anal & Dolor al defecar \\
Aumento de la fermentación y del meteorismo & Dolor crónico visceral \\
Efecto central de los opioides & Náuseas y vómitos \\
\hline
\end{tabular}


Tabla II. Criterios diagnósticos del estreñimiento inducido por opioides basados en el consenso Roma IV ${ }^{2}$

A. Las deposiciones ligeras no están presentes sin el uso de laxantes

B. Síntomas de estreñimiento nuevos o que empeoran al iniciar, cambiar o aumentar la terapia con opioides y que debe incluir dos o más de los siguientes:

1. Heces grumosas o duras en más de un $25 \%$ de las defecaciones

2. Esfuerzo durante más de un $25 \%$ de las defecaciones

3. Sensación de evacuación incompleta en más de un $25 \%$ de las defecaciones

4. Sensación de obstrucción o bloqueo anorrectal durante más de un $25 \%$ de las defecaciones

5. Maniobras manuales para facilitar la evacuación en más de un $25 \%$ de las defecaciones, como evacuación digital o presión del piso pélvico

6. Menos de tres deposiciones espontáneas por semana

por un médico y reportado por el paciente, de tres ítems para evaluar el EIO en pacientes con dolor crónico. Incluye tres variables: "facilidad de defecación", "sensación de evacuación intestinal incompleta" y "juicio personal global de estreñimiento". Los pacientes clasifican estas variables según su experiencia durante los 7 días anteriores, mediante una escala analógica numérica de 0-100 donde 0 representa la ausencia del síntoma y 100 denota la dificultad máxima o el signo más grave. La puntuación IFI se calcula como la media de las puntuaciones de los tres componentes. El rango de referencia IFI, para pacientes no estreñidos con dolor crónico, se puede definir entre 0 y 28,8 según los estudios publicados ${ }^{7}$. El IFI ha sido validado psicométricamente, responde a los cambios en la gravedad de los síntomas y es una herramienta de evaluación simple con un umbral aprobado de estreñimiento clínicamente significativo; por lo tanto, basado en el consenso internacional, los tratamientos de prescripción para EIO deben considerarse para pacientes que tienen una puntuación IFI igual o superior a 30 puntos y una respuesta inadecuada a intervenciones de primera línea (dieta, laxantes, ejercicio) $^{8}$.

3. Para considerar que el paciente tenía una respuesta insuficiente a los laxantes fue necesario que en las 2 semanas previas a la primera visita del estudio hubiera informado de varios síntomas simultáneos de EIO. Estos últimos debían ser de intensidad moderada mientras recibía al menos una clase de laxantes durante un periodo mínimo de 7 días antes del estudio.

\section{Recomendaciones de uso de naloxegol para los pacientes del estudio}

La dosis inicial recomendada de naloxegol para tratar el EIO debía ser siempre una píldora diaria de $25 \mathrm{mg}$ antes del desayuno sin laxantes, según lo recomendado en la ficha técnica?.

\section{Método de valoración}

El método fue el siguiente:

A todos los pacientes se les debía recetar media dosis si presentaban efectos adversos digestivos moderados durante la primera semana. Por lo tanto, debían cambiar a $12,5 \mathrm{mg} /$ día.

A los pacientes que necesitasen ajustar la dosis a una dosis inferior a 12,5 mg/día debido a efectos secundarios gastrointestinales graves, se les permitía usar una píldora de $12,5 \mathrm{mg} /$ día cada 2 días.

Si este último cambio no mejoraba el EIO, o también presentaban efectos secundarios intolerables, podrían suspender su participación en el estudio o reanudar los laxantes. Los tres pacientes completaron el estudio.

- Los pacientes fueron seguidos de cerca de través de una llamada telefónica diaria de la enfermería durante la primera semana $y$, después, una vez por semana desde el inicio del tratamiento hasta alcanzar una dosis efectiva.

- Los pacientes fueron revisados en una consulta médica en la primera semana (7 días), al primer mes (30 días), al tercer mes (90 días), al sexto mes (180 días) y al año (365 días).

Se evaluaron cinco parámetros:

Objetivos primarios. El principal objetivo fue el número de movimientos intestinales espontáneos, con una tasa de respuesta durante el periodo de tratamiento de 6 meses definida como tres o más por semana. El IFI, que también se midió al comienzo, a los 6 meses y al año, se consideró también objetivo primario.

Objetivos secundarios. Se consideraron los siguientes:

- Mejoría en la escala de tipos de heces, medida por la escala de heces de Bristol (Bristol Stool Form Scale, BSFS) de 1 (peor) a 7 (mejor). Los pacientes con heces correspondientes al tipo 1 -que se definen como excrementos de conejos (pequeños, duros y grumosos, que parecen heces de cabra) - y tipo 2 -definidas como racimos (varios bultos duros que parecen heces de cabra y racimos) - se consideraron estreñidos. Los que tenían heces de tipo 3 (maíz) y 4 (salchicha) se consideran normales, el tipo 5 (nuggets de pollo) como carente de fibra y los tipos 6 (avena) y 7 (salsa) como inflamación ${ }^{10}$.

- Finalmente, se realizó una valoración mediante la escala visual analógica de dolor (EVA) de 0 (mejor) a 10 (peor), y de la incidencia de efectos adversos. Para medir la satisfacción personal se usó una escala de cinco niveles (satisfacción muy baja, baja, normal, alta, muy alta).

\section{Pacientes}

- Paciente 1. Varón de 72 años de edad con dolor como resultado de una fractura por compresión vertebral doble a nivel de las vértebras torácicas $10^{\mathrm{a}}$ y $12^{\mathrm{a}}$, relacionada con la metástasis de un mieloma múltiple. 
El dolor basal se controlaba con un parche de fentanilo transdérmico de $50 \mu \mathrm{g} / \mathrm{h}$ cada 3 días, naproxeno $500 \mathrm{mg} / 12 \mathrm{~h}$, paracetamol $1 \mathrm{~g} / 8 \mathrm{~h}$ y clonazepam $0,5 \mathrm{mg}$ por la noche. El dolor irruptivo incidental del paciente se controlaba con fentanilo intranasal en gel de pectina $100 \mu \mathrm{g}$ como régimen de rescate. El EIO se trataba con lactulosa $15 \mathrm{ml}$ dos veces al día, dieta rica en fibra y ejercicio aeróbico diario.

- Paciente 2. Varón de 65 años con dolor en el tórax relacionado con metástasis pleurales en el contexto de dolor por cáncer de pulmón. También presentó dolor neuropático postoracotomía crónica. El dolor basal estaba bien controlado con oxicodona/naloxona de liberación retardada $20 / 10 \mathrm{mg}$ cada $12 \mathrm{~h}$, paracetamol $1 \mathrm{~g} / 8 \mathrm{~h}$, pregabalina $75 \mathrm{mg} / 12 \mathrm{~h}$ y $75 \mathrm{mg}$ de amitriptilina por la noche. El dolor irruptivo ocasional se mejoró a un nivel tolerable para su actividad (EVA < 3) con una dosis diaria de $200 \mu \mathrm{g}$ de aerosol nasal de fentanilo en pectina. El EIO se trató con lactulosa $15 \mathrm{ml}$ dos veces al día y senna $15 \mathrm{mg}$ una vez al día, dieta rica en fibra y ejercicio aeróbico diario.

- Paciente 3. Mujer de 47 años con dolor lumbar debido a una metástasis vertebral en la $5^{\text {a }}$ dorsal con cáncer de mama subyacente. El dolor basal se controló con oxicodona/naloxona $40 / 20$ cada 12 h, naproxeno
$500 \mathrm{mg} / 12 \mathrm{~h}$, paracetamol $1 \mathrm{~g} / 8 \mathrm{~h}$ y diazepam $10 \mathrm{mg}$ por noche. El dolor irruptivo se mejoró (EVA < 3) con aerosol nasal de fentanilo en pectina, con una de $200 \mu \mathrm{g} / 12 \mathrm{~h}$. El ElO se trató con senna $30 \mathrm{mg}$ una vez al día y macrogol 2 sobres al día, dieta rica en fibra y ejercicio aeróbico diario.

\section{Resultados}

Los principales resultados demostraron que el tratamiento con naloxegol mejoró, en todos los pacientes, los resultados primarios, como IFI inferior a 30 , y también el número de deposiciones por semana, sin afectar a sus hábitos dietéticos y al plan de vida recomendado, que mantuvieron durante el estudio. La escala BSFS también mejoró en todos ellos. El nivel de analgesia, medido por la escala EVA, no cambió a lo largo del estudio, y la satisfacción personal se definió al final de la encuesta como alta/muy alta. El punto clave fue la dosis de naloxegol y la frecuencia seleccionada por los pacientes en relación con los efectos secundarios abdominales. Asimismo, dos de los pacientes comunicaron que usaban laxantes ocasionalmente como ayuda puntual para no interferir en los resultados globales, que se resumen en la Tabla III.

Tabla III. Resultados del estudio observacional de tres casos de 1 año de naloxegol en pacientes con cáncer

\begin{tabular}{|c|c|c|c|}
\hline & Paciente 1 & Paciente 2 & Paciente 3 \\
\hline EVA basal & $<3$ & $<3$ & $<3$ \\
\hline Opioide basal & $\begin{array}{l}\text { Fentanilo transdérmico } \\
50 \mathrm{mg} / \mathrm{h} / 72 \mathrm{~h}\end{array}$ & $\begin{array}{l}\text { Oxicodona/naloxona } \\
20 / 10 \mathrm{mg} / 12 \mathrm{~h}\end{array}$ & $\begin{array}{l}\text { Oxicodona/naloxona } \\
40 / 20 \mathrm{mg} / 12 \mathrm{~h}\end{array}$ \\
\hline Opioide para dolor irruptivo & $\begin{array}{c}\text { Fentanilo intranasal } \\
100 \mathrm{mg}\end{array}$ & $\begin{array}{l}\text { Fentanilo intranasal } \\
200 \mathrm{mg}\end{array}$ & $\begin{array}{l}\text { Fentanilo intranasal } \\
200 \mathrm{mg}\end{array}$ \\
\hline Tratamiento laxante del EIO & Lactulosa $15 \mathrm{ml} / 12 \mathrm{~h}$ & $\begin{array}{l}\text { Lactulosa } 15 \mathrm{ml} / 12 \mathrm{~h} \text { y } \\
\text { senna } 15 \mathrm{mg} / 24 \mathrm{~h}\end{array}$ & $\begin{array}{c}\text { Macrogol } 2 \text { sobres } / 24 \mathrm{~h} \text { y } \\
\text { senna } 30 \mathrm{mg} / 24 \mathrm{~h}\end{array}$ \\
\hline $\mathrm{IFI}$ al inicio del estudio & 75 & 73 & 81 \\
\hline $\begin{array}{l}\text { Movimientos intestinales } \\
\text { espontáneos/semana } \\
\text { al inicio del estudio }\end{array}$ & 1 & 2 & 2 \\
\hline Escala de Bristol al inicio del estudio & 1 & 2 & 2 \\
\hline Dosis de naloxegol al final de estudio & $12,5 \mathrm{mg} / 48 \mathrm{~h}$ & $12,5 \mathrm{mg} / 24 \mathrm{~h}$ & $25 \mathrm{mg} / 24 \mathrm{~h}$ \\
\hline $\begin{array}{l}\text { Movimientos intestinales } \\
\text { espontáneos/semana al final del } \\
\text { estudio }\end{array}$ & 4 & 7 & 7 \\
\hline Escala de Bristol al final del estudio & 3 & 4 & 5 \\
\hline IFI al final del estudio & 25 & 23 & 21 \\
\hline Efectos adversos & $\begin{array}{l}\text { Dolor abdominal intenso } \\
\text { con dosis } 25 \mathrm{mg} / 24 \mathrm{~h} \text { y } \\
\text { moderado con } 12,5 \mathrm{mg} / \\
\text { día }\end{array}$ & $\begin{array}{l}\text { Dolor abdominal } \\
\text { moderado con dosis } \\
25 \mathrm{mg} / 24 \mathrm{~h}\end{array}$ & $\begin{array}{l}\text { Dolor abdominal leve con } \\
\text { dosis } 25 \mathrm{mg} / 24 \mathrm{~h}\end{array}$ \\
\hline EVA al final del estudio & $<3$ & $<3$ & $<3$ \\
\hline Satisfacción global & Buena & Muy buena & Muy buena \\
\hline
\end{tabular}

EIO: estreñimiento inducido por opioides. EVA: escala visual analógica del dolor. IFI: índice de función intestinal. 


\section{Consideraciones finales}

El naloxegol es un derivado pegilado de la molécula de naloxona. La pegilación o unión de un polietilenglicol (PEG) a un fármaco genera un derivado muy estable, que se caracteriza por presentar una gran velocidad de absorción y una eliminación que condiciona la persistencia de concentraciones plasmáticas durante un periodo de tiempo efectivo. Este proceso evita que cruce la barrera hematoencefálica debido al aumento del tamaño de la partícula, así como a la reducción de la permeabilidad pasiva y activa.

La eficacia global de los fármacos PAMORA es actualmente objeto de debate y motivo de controversia. Una revisión sistemática y un metaanálisis ${ }^{10}$ mostraron que estos fármacos eran más efectivos que el placebo en el tratamiento del EIO, con números necesarios para tratar de 3,4 y 5 para metilnaltrexona, naloxona y alvimopán, respectivamente. El alvimopán solo está aprobado para el periodo postoperatorio y no puede utilizarse para tratar el EIO. La metilnaltrexona es un compuesto de amonio cuaternario, y por tanto extremadamente polar, que no atraviesa fácilmente las membranas biológicas, tales como las células epiteliales en el intestino y las células endoteliales en la barrera hematoencefálica. Por ello, el medicamento no tiene acceso al sistema nervioso central y debe administrarse por vía parenteral. Es bien tolerado por todos los pacientes, pero su alto precio y vía de administración subcutánea limitan su uso rutinario ${ }^{11}$.

En una revisión sistemática del Grupo de Trabajo Nórdico sobre la eficacia de PAMORA en el tratamiento del $\mathrm{EIO}^{3}$, se concluyó, de acuerdo con el sistema de revisión GRADE (Grading of Recommendations Assessment, Development and Evaluation), lo siguiente:

El naloxegol bloquea los receptores opioides periféricos y se puede utilizar para minimizar el ElO (evidencia de calidad moderada, recomendación fuerte).

- La metilnaltrexona bloquea los receptores opioides periféricos y puede usarse para minimizar el EIO (evidencia de calidad moderada, recomendación débil).

Finalmente, una revisión sistemática publicada en $2017^{12}$ concluyó que los antagonistas del receptor $\mu$ opioide de acción periférica pueden ser útiles en el tratamiento de la DIIO, pero las conclusiones definitivas para su recomendación clínica no fueron posibles debido a la incoherencia de los estudios revisados y la calidad de la evidencia relativamente baja. La comparación de los agentes no fue razonable debido a criterios de valoración no homogéneos o la ausencia de estudios comparativos.

La DIIO es un problema creciente en relación con el incremento en la prescripción de este grupo de fármacos. Sin embargo, no debe pasarse por alto que hay muchas otras razones para el estreñimiento que también deben tenerse en cuenta en el diagnóstico de cada paciente individualmente. Los laxantes se recomiendan como medicamentos de primera línea. Los cambios en el estilo de vida y analgésicos alternativos siempre deberían considerarse. La disminución de la dosis de opioides, la rotación a oxicodona/naloxona y los fármacos de acción dual como el tapentadol también pueden mejorar la DIIO. Cuando las medidas anteriores no logran aliviar los síntomas, se debe considerar el tratamiento con PAMORA. Posteriormente, el tratamiento optimizado mejorará la calidad de vida y, desde una perspectiva socioeconómica, también puede reducir los costes asociados ${ }^{13}$. Nuestro estudio clínico respalda la consideración de que el naloxegol proporciona un beneficio terapéutico sobre los laxantes, pero creemos que estos no deberían ser retirados obligatoriamente, especialmente en pacientes con cáncer y con EIO más grave, ya que al presentar un mecanismo de acción diferente podrían ayudar a disminuir la dosis de naloxegol, o mejorar el cuadro clínico en pacientes con pauta fija en dosis altas ( $25 \mathrm{mg} /$ día). Finalmente, se podría recomendar comenzar con la dosis mínima eficaz $(12,5 \mathrm{mg} /$ día) y reservar las dosis altas ( $25 \mathrm{mg} /$ día $)$ para aquellos pacientes que toman opioides en mayor dosis.

Respecto a la conclusión final, dado que el soporte de la bibliografía es relativamente pobre y el número de casos incluido en el estudio pequeño, debería considerarse que se abre un nuevo grupo farmacológico para el manejo del estreñimiento, en el que necesitamos tener más experiencia y manejo para conocer su eficacia, y, en cualquier caso, hemos de ir evaluando qué lugar ocupan en el manejo global del EIO.

\section{Bibliografía}

1. Pappagallo $M$. Incidence prevalence, and management of opioid bowel dysfunction. Am J Surg. 2001;182(5A Suppl):11S-18S.

2. Simren M, Palsson OS, Whitehead WE. Update on Rome IV Criteria for Colorectal Disorders: Implications for Clinical Practice. Curr Gastroenterol Rep. 2017;19:15.

3. Drewes AM, Munkholmb P, Simrénc M, Breivikd H, Kongsgaarde UE, Hatlebakkf JG, et al. Definition, diagnosis and treatment strategies for opioid-induced bowel dysfunction. Recommendations of the Nordic Working Group. Scandin J Pain. 2016;11:111-22.

4. Bell TJ, Panchal SJ, Miaskowski C, Bolge SC, Milanova T, Williamson R. The prevalence, severity, and impact of opioid-induced bowel dysfunction: results of a US and European Patient Survey (PROBE 1). Pain Med. 2009;10:35-42.

5. Dennison C, Prasad M, Lloyd A, Bhattacharyya SK, Dhawan R, Coyne K. The health-related quality of life and economic burden of constipation. Pharmacoeconomics. 2005;23:461-76.

6. Hjalte F, Berggren AC, Bergendahl H, Hjortsberg C. The direct and indirect costs of opioid-induced constipation. J Pain Symptom Manage. 2010;40:696-703.

7. Ueberall MA, Müller-Lissner S, Buschmann-Kramm C, Bosse B. The Bowel Function Index for evaluating constipation in pain patients: definition of a reference range for a non-constipated population of pain patients. J Int Med Res. 2011;39:41-50.

8. Argoff CE, Brennan MJ, Camilleri M, Davies A, Fudin J, Galluzzi $\mathrm{KE}$, et al. Consensus Recommendations on Initiating Prescription Therapies for Opioid-Induced Constipation. Pain Med. 2015; 16:2324-37.

9. Agencia Española de Medicamentos y Productos Sanitarios. Informe de posicionamiento terapéutico PT-NALOXEGOL/ V1/27102016. [Consultado el 24 de marzo de 2018]. Disponible en: https://www.aemps.gob.es/medicamentosUsoHumano/informesPublicos/docs/IPT-naloxegol-Moventig-estrenimiento.pdf

10. Ford AC, Brenner DM, Schoenfeld PS. Efficacy of pharmacological therapies for the treatment of opioid-induced constipation: systematic review and metaanalysis. Am J Gastroenterol. 2013;108:1566-74.

11. Chey WD, Webster L, Sostek M, Lappalainen J, Barker PN, Tack J. Naloxegol for opioid-induced constipation in patients with noncancer pain. N Engl J Med. 2014;370:2387-96.

12. Schwenk ES, Grant AE, Torjman MC, McNulty SE, Baratta JL, Viscusi ER. The efficacy of peripheral opioid antagonists in opioidinduced constipation and postoperative ileus: a systematic review of the literature. Reg Anesth Pain Med. 2017;42:767-77.

13. Leppert W. Emerging therapies for patients with symptoms of opioid-induced bowel dysfunction. Drug Des Devel Ther. 2015; 16:2215-31. 